\title{
O ELETRÓLITO SUPORTE E SUAS MÚLTIPLAS FUNÇÕES EM PROCESSOS DE ELETRODO
}

Silvia M. L. Agostinho* e Ruth F. V. Villamil

Instituto de Química, Universidade de São Paulo, Av. Prof. Lineu Prestes, 748, 05508-900 São Paulo - SP

Augusto Agostinho Neto

Departamento de Física, Instituto de Biociências, Letras e Ciências Exatas, Universidade Estadual Paulista "Júlio de Mesquita Filho", São José do Rio Preto - SP

Hernani Aranha

Universidade Metropolitana de Santos, Rua da Constituição, 374, 11015-470 Santos - SP e Núcleo de Ciências Biológicas e da Saúde, Centro Universitário das Faculdades Metropolitanas Unidas, Av. Santo Amaro, 1239, 04505-002 São Paulo - SP

Recebido em 5/8/03; aceito em 18/2/04; publicado na web em 17/6/04

\begin{abstract}
THE SUPPORTING ELECTROLYTE AND ITS MULTIPLE FUNCTIONS ON ELECTRODE PROCESSES. The aim of this work is to present the principal properties and applications of supporting electrolytes (SE) to students, teachers and researchers interested in electrode processes. Different aspects are discussed including the importance of SE in maintaining constant the activity coefficients and the diffusion coefficients and reducing the transport number of electroactive species. Its effect on the electrochemical kinetic parameters is also presented.
\end{abstract}

Keywords: electrochemistry; supporting electrolyte; electrode processes.

\section{INTRODUÇÃo}

O eletrólito suporte é utilizado com frequência por pesquisadores e professores que se dedicam a estudos de interfaces metal-solução eletrolítica. A sua importância pode ser encontrada na abordagem de aspectos termodinâmicos, na descrição de modelos e no estudo de cinética eletroquímica, nas propriedades elétricas de eletrólitos e em transporte de massa em soluções eletrolíticas ${ }^{1-14}$. O que se constata, entretanto, é a quase ausência, na literatura, de um artigo ou capítulo de livro em que se discuta a larga aplicabilidade do eletrólito suporte, bem como os cuidados em sua escolha. Exceção se faz ao capítulo descrito por Sawyer et al. ${ }^{10}$, em seu livro Experimental Electrochemistry for Chemists, onde são enfatizados aspectos eletroanalíticos.

Este trabalho é dirigido a todos aqueles que se dedicam ao ensino de graduação e à pesquisa nas áreas de Eletroquímica e Eletroanalítica, e, em particular, aos estudantes que fazem iniciação científica ou pós-graduação nestas áreas.

Alguns conceitos relacionados ao tema deste trabalho, para não alongar o texto, não foram abordados detalhadamente, mas podem ser encontrados nas referências citadas. Admite-se que o leitor, para um bom entendimento do artigo, esteja familiarizado com fundamentos de termodinâmica química ${ }^{15,16}$, da dupla camada elétrica ${ }^{1-5}$, da cinética eletroquímica ${ }^{1-14}$, da condução iônica ${ }^{15,16}$ e do transporte de massa em eletrólitos ${ }^{4,5,8}$.

Este trabalho tem como objetivos conceituar o eletrólito de suporte, apresentar sugestões sobre a escolha adequada do mesmo, enumerar e discutir suas múltiplas funções e dar exemplos ilustrando os efeitos de sua adição a sistemas eletroquímicos. Alguns destes exemplos podem servir de sugestões a educadores na utilização em laboratórios didáticos ou experimentos demonstrativos.

É importante salientar que este trabalho se refere a eletrodos com dimensões convencionais, isto é, superiores a $50 \mu \mathrm{m}$. A discussão não incluirá o emprego de microeletrodos ou ultramicroeletrodos.

*e-mail: smlagost@iq.usp.br
Neste caso, as densidades de corrente muito elevadas geram, na vizinhança do eletrodo, devido aos processos faradaicos, uma variação muito grande na concentração de íons. Em consequiência, o papel desempenhado pelo eletrólito suporte precisa ser tratado de forma diferente. Recomenda-se aos leitores interessados que recorram a referências como os trabalhos de Oldham ${ }^{17} \mathrm{e}$ de Hyk et al. ${ }^{18}$.

\section{O QUE É UM ELETRÓLITO?}

Uma definição considerada apropriada para eletrólito é proposta por Lobo ${ }^{19}$ : "Eletrólito é uma substância que, quando dissolvida em um dado solvente, produz uma solução com uma condutividade elétrica maior que a condutividade do solvente". Considerando como solvente a água, servem de exemplos como eletrólitos: sais (cloreto de sódio), ácidos (ácido sulfúrico) e bases (hidróxido de sódio).

\section{O QUE É UM ELETRÓLITO SUPORTE?}

Em sistemas eletroquímicos o eletrólito suporte (ou eletrólito de suporte) é um eletrólito que, adicionado em altas concentrações (cerca de cem vezes maior que a da espécie eletroativa $)^{14}$, pode conferir à solução e à interface (do tipo metal-solução) em estudo uma série de propriedades. Tais propriedades, em geral são resultantes da manutenção da força iônica alta e constante da solução, o que, como será visto, simplifica a análise dos sistemas eletroquímicos.

\section{COMO ESCOLHER ADEQUADAMENTE O ELETRÓLITO SUPORTE?}

O eletrólito suporte deve, em princípio, apresentar as seguintes características: alta solubilidade, alto grau de ionização e ser estável química e eletroquimicamente no solvente a ser empregado.

Entenda-se por estabilidade química do eletrólito suporte suas espécies químicas não reagirem com outras espécies presentes na solução (eletroativas ou não), enquanto a estabilidade eletroquímica implica em admitir que o eletrólito não seja oxidado nem reduzido 
eletroliticamente em toda a faixa de potencial de interesse a que as interfaces em estudo forem submetidas.

Em meios aquosos são muito empregados, como eletrólito suporte, sais que, além das propriedades acima mencionadas, não apresentem hidrólise significativa e nem formem complexos com os íons em solução. Podem ser citados, em meio aquoso, os percloratos, nitratos e sulfatos de sódio e de potássio. Os cloretos de metais alcalinos podem ser utilizados, mas é preciso lembrar a forte tendência dos íons cloreto em formar complexos com íons metálicos, em particular, os de metais de transição ${ }^{20}$. Em meios aquosos ácidos destacam-se, como eletrólito suporte, os ácidos perclórico, sulfúrico e nítrico, além do ácido clorídrico, com as restrições acima citadas. Em meios aquosos alcalinos dá-se preferência aos hidróxidos de sódio e de potássio. Alguns autores consideram eletrólito suporte um sistema tamponante como, por exemplo, citrato, fosfato, acetato, borato, quando se faz necessário manter constante, além da força iônica, o $\mathrm{pH}$ da solução aquosa ${ }^{11}$.

Em meios orgânicos de acetona, acetonitrila, dimetilformamida, dimetilsulfóxido, diclorometano, empregam-se, como eletrólito suporte, sais quaternários de amônio, por exemplo, brometo, tetrafluorborato ou perclorato de tetraetil ou de tetrabutil amônio. Sawyer et al. ${ }^{10}$ apresentam as propriedades de vários sais de alquil amônio, entre elas, solubilidade, resistividade e condutividade à diluição infinita em diferentes solventes orgânicos.

\section{AS MÚLTIPLAS FUNÇÕES DO ELETRÓLITO SUPORTE}

\section{Manter os coeficientes de atividade das espécies eletroativas praticamente constantes}

Em sistemas não ideais (que não obedecem à lei de Raoult), por exemplo, em soluções eletrolíticas, o potencial químico, ou energia livre molar da espécie $i\left(\mu_{i}\right)$, depende da atividade $a_{i}$ desta espécie, de acordo com a Equação 1,

$\mu_{i}=\mu_{i}^{\circ}+R T \ln a_{i}$

onde $\mu_{i}^{\circ}$ é o potencial químico padrão à temperatura $T$ e $R$, a constante universal dos gases.

Se a espécie $i$ for um íon, o potencial eletroquímico $\tilde{\mu}_{i}$ é dado pela Equação 2,

$\tilde{\mu}_{i}=\mu_{i}+z_{i} F \psi$

onde $\psi$ representa o potencial elétrico a que o íon está submetido; $z_{i}$ é a carga do íon e $F$, a constante de Faraday.

As soluções eletrolíticas afastam-se significativamente da idealidade. Nestas condições, a atividade $a_{i}$ da espécies $i$ presente em solução depende não linearmente da sua concentração $\left(C_{i}\right)$.

A definição operacional de atividade é dada pela Equação 3,

$a_{i}=\gamma_{i} C_{i}$

onde $\gamma_{i}$, o coeficiente de atividade da espécie “ $i$ ”, descreve a interação iônica e o desvio da idealidade, e pode ser expresso como uma função polinomial da concentração.

$\mathrm{Na}$ ausência de eletrólito suporte, à medida que as espécies eletroativas sofrem redução ou oxidação na interface eletrodo-solução, as concentrações mudam em suas vizinhanças e, em conseqüência, o valor de $\gamma_{i}$. A presença do eletrólito suporte em concentração muito maior que a da espécie " $i$ " faz com que o número de vizinhos de carga contrária em torno do íon central (eletroativo) se torne praticamente constante durante o processo de eletrodo tornan- do, em consequiência, as interações iônicas constantes e $\gamma_{i}$ independente de $C_{i}$.

Um exemplo prático. Cálculo do potencial de eletrodo. Consideremos a equação representativa do equilíbrio $\mathrm{Cu}(\mathrm{II}) / \mathrm{Cu}$

$C u^{2+}+2 e^{-} \Leftrightarrow C u$

$\mathrm{Na}$ ausência de eletrólito suporte o potencial de redução $E_{\mathrm{Cu}^{2+} / \mathrm{Cu}^{2+}}$ é dado pela equação de Nernst.

$E_{\mathrm{Cu}^{2+} / \mathrm{Cu}}=E_{\mathrm{Cu}^{2+} / \mathrm{Cu}}^{\circ}+\frac{R T}{2 F} \ln \left[a_{\mathrm{Cu}^{2+}} / a_{\mathrm{Cu}}\right]$

onde $E_{\mathrm{Cu}^{2+} / \mathrm{Cu}}^{\circ}$ é o potencial de redução padrão; $R$, a constante universal dos gases; $T$, a temperatura absoluta; $F$, o número de Faraday e $a_{\mathrm{Cu}^{2+}}$ e $a_{C u}$ são as atividades do íon $C u^{2+}$ e do cobre metálico, respectivamente.

Admitindo o cobre como metal puro, $a_{C u}=1$. Na presença de eletrólito suporte, $\gamma_{\mathrm{Cu}^{2+}}$ é aproximadamente constante e pode-se escrever.

$E=E^{\circ}+\frac{R T}{2 F} \ln C_{\mathrm{Cu}^{2+}}$

onde $E^{\circ}=E^{\circ}+\frac{R T}{2 F} \ln \gamma_{C u^{2+}}$

$E^{\circ \prime}$ representa o potencial de redução padrão formal para a reação $C u^{2+} / C u$ em meio contendo um determinado eletrólito suporte na concentração escolhida e à temperatura $T$.

A presença do eletrólito suporte, portanto, permite estimar o valor do potencial do eletrodo como uma função da concentração. Podese mostrar que a força eletromotriz da pilha de Daniel é uma função linear do $\ln \left(C_{\mathrm{Zn}^{2+}} / C_{\mathrm{Cu}^{2+}}\right)$, empregando como eletrólito suporte o sulfato de sódio ${ }^{21}$.

É importante salientar que enquanto $E^{\circ}$, o potencial padrão, depende da natureza da interface condutor eletrônico-condutor iônico e da temperatura, o potencial padrão formal $E^{\circ}$ depende, além destes fatores, da natureza e da concentração do eletrólito suporte.

\section{Tornar o número de transporte da espécie eletroativa praticamente igual a zero}

Uma corrente elétrica, numa solução eletrolítica, é o resultado das contribuições do movimento orientado de diferentes espécies carregadas. No caso da corrente contínua, a corrente é devida ao movimento de translação de cátions e ânions em sentidos contrários, sob ação do campo elétrico.

O número de transporte de um íon $i$ corresponde à fração de corrente devida ao mesmo, sendo dado pela Equação 6.

$t_{i}=\frac{I_{i}}{I}$

onde $I_{i}$ é a corrente devida ao movimento orientado dos ions $i$ e $I$, a corrente iônica resultante.

Esta fração é tão maior quanto maior a concentração da espécie $i$, uma vez que a contribuição à corrente $I_{i}$, é dada pela Equação 7 .

$I_{i}=C_{i} v_{i} z_{i} F A$

onde $C_{i}$ é a concentração da espécie $i$ carregada (íon); $v_{i}$, a velocidade do íon $i ; z_{i}$, a carga do íon $i$ e $A$ é a área da seção transversal do eletrodo. 
A adição de eletrólito suporte (ES) faz com que o valor de $t$ tenda a zero para a espécie eletroativa, se $C_{i}<<C_{E S}$, onde $\mathrm{C}_{\mathrm{ES}}$ é a concentração do eletrólito suporte. Nestas condições, o transporte de massa desse íon à superfície ou a partir da superfície do eletrodo em que ocorrerá a reação se dará apenas por difusão (em solução termostatizada e sem convecção forçada) ou por difusão e convecção, se houver movimento macroscópico do fluido.

A Tabela 1 ilustra o efeito do eletrólito suporte $\mathrm{KNO}_{3}$ sobre a corrente limite $\left(I_{L}\right)$ do íon $P b^{2+}$, quando reduzido sobre eletrodo de mercúrio gotejante ${ }^{22}$, de área constante.

Tabela 1. Efeito do eletrólito suporte sobre a corrente limitante $\left(I_{L}\right)$

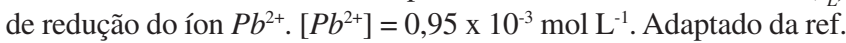
22

\begin{tabular}{cc}
\hline$\left[\mathrm{KNO}_{3}\right] / \mathrm{mol} \mathrm{L}^{-1}$ & $I_{L} / \mu A$ \\
\hline 0 & 17,6 \\
0,005 & 9,8 \\
0,1 & 8,45 \\
1,0 & 8,45 \\
\hline
\end{tabular}

Observa-se que a corrente decresce com a adição de $\mathrm{KNO}_{3}$, até que, para concentração de $\mathrm{KNO}_{3}$ maior ou igual a $0,1 \mathrm{~mol} \mathrm{~L}^{-1}$, se mostra constante. Como o $\mathrm{Pb}^{2+}$, a espécie a ser reduzida, é um cátion, quando não há eletrólito suporte o valor de $t_{i}$ é mais elevado, devido à presença do transporte por migração (ou condução) causado por um $t_{i}$ significativo, além do transporte difusivo-convectivo à gota de mercúrio, já existente.

$\mathrm{Na}$ redução do íon dicromato $\mathrm{Cr}_{2} \mathrm{O}_{7}^{2-}$, o ES exerce um efeito inverso sobre o valor de $I_{L}$ por se tratar de um ânion; o campo elétrico o faz migrar para o ânodo, levando a valores de $I_{L}$ menores, na ausência do ES ou na sua presença em concentrações pouco significativas (quando os números de transporte de $i$ e das espécies presentes no ES são comparáveis).

\section{Diminuir a espessura da dupla camada elétrica}

A interface metal-solução pode ser descrita através do modelo da dupla camada elétrica, constituída de uma camada compacta (próximo ao eletrodo) e uma camada difusa (em direção ao meio da solução) conforme esquematizada na Figura 1. Na ausência de adsorção específica, o plano correspondente à espessura $x_{1}$ passa pelo centro das moléculas do solvente e recebe o nome de plano interno de Helmholtz $(\mathrm{PIH})$, enquanto o plano correspondente a $\mathrm{x}_{2}$ passa pelo centro dos íons solvatados mais próximos, de carga contrária à da superfície metálica e recebe o nome de plano externo de Helmholtz (PEH) (Figura 1a). No caso de adsorção específica, o PIH passa pelo centro dos íons especificamente adsorvidos, conforme se vê na Figura 1b. Além do $\mathrm{PEH}$, isto é, para $\mathrm{x} \geq \mathrm{x}_{2}$ estende-se a camada difusa, onde o potencial $\phi(\mathrm{x})$ varia até atingir o valor de $\phi_{\mathrm{s}}$, isto é, o potencial no meio da solução.

Na camada difusa a distribuição de concentrações $C$ das espécies carregadas, bem como o potencial $\phi$, podem ser descritos pelas equações Poisson-Boltzmann e dependem da coordenada posição x, em relação ao eletrodo ${ }^{1-14}$.

O potencial $\phi(x)$ para $x \geq x_{2}$ pode ser dado pela Equação $8^{23}$ :

$\phi\left(x^{\prime}\right)= \pm\left(\frac{4 R T}{z F}\right) \tanh ^{-1}\left\{\left(\tanh \left|\frac{z F \phi_{2}}{4 R T}\right|\right) e^{-\kappa x^{\prime}}\right\}$

a variável x' é dada por $x^{\prime}=x_{-}-x_{2} ; x_{2} \geq x_{2}$. As outras grandezas têm o seguinte significado: $\mathrm{R}$ é a constante universal dos gases, $\mathrm{T}$ a tempe-

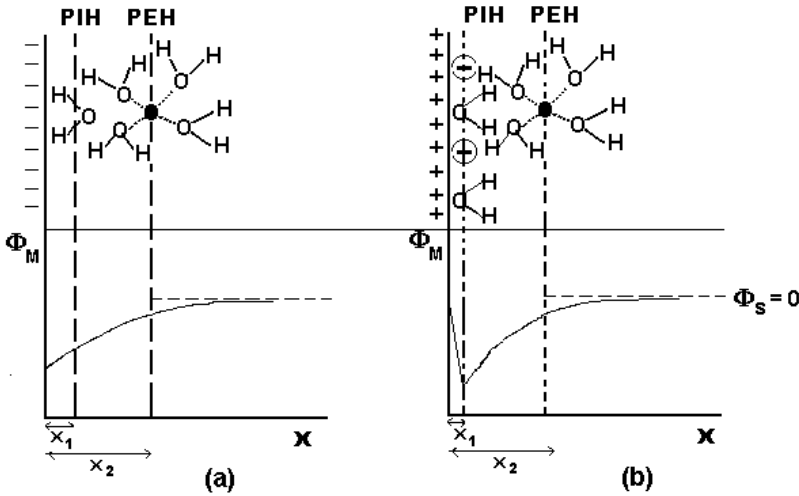

Figura 1. Visão qualitativa da dupla camada elétrica na ausência (a) e presença (b) de adsorção especifica. PIH - Plano interno de Helmholtz; PEH - Plano externo de Helmhotz; $x_{1}$ - espessura da camada interna. $x_{2}$-espessura da camada externa; - cátion; $\Phi_{M}$-potencial no metal; $\odot$ - ânion especificamente adsorvido; $\Phi_{s}$ - potencial no meio da solução

ratura absoluta, $\mathrm{z}_{\mathrm{i}}$ a carga do íon, $\mathrm{F}$ a constante de Faraday, $\phi_{2}$ é o potencial no PEH e $\kappa$ é o inverso do comprimento de Debye.

A concentração da espécie iônica i, para $\mathrm{x} \geq \mathrm{x}_{2}$, é dada pela Equação 9:

$C_{i}(x)=C_{i}^{\circ} \exp \left[\frac{-z_{i} F \phi(x)}{R T}\right]$

onde $C_{i}$ é a concentração da espécie $i$ no meio da solução (a uma distância infinita do eletrodo), onde se admite $\phi=\phi_{\mathrm{s}}=0$.

A Tabela 2 ilustra a espessura efetiva da camada difusa $(\delta)$ para eletrólitos 1:1, isto é, em que as cargas do cátion e do ânion são iguais à unidade, como uma função da concentração do eletrólito. $\mathrm{O}$ valor de $\delta$ corresponde à região próxima ao eletrodo, dentro da qual ocorre $99,99 \%$ da perturbação de potencial, calculada a partir do modelo de Gouy-Chapman. Em outras palavras, corresponde à espessura da camada difusa.

A Tabela 2 serve para ilustrar o efeito significativo do eletrólito suporte, no sentido de reduzir a espessura da dupla camada elétrica. A concentração maior empregada torna o valor de $\delta$ igual a $28 \AA$, aproximando-o das dimensões moleculares.

Tabela 2. Espessura efetiva da camada difusa para um eletrólito 1:1 a $25{ }^{\circ} \mathrm{C}$. Adaptado da ref. 23

\begin{tabular}{cc}
\hline Concentração do eletrólito/mol L & \multicolumn{1}{|c}{$\delta / \mathrm{cm}$} \\
\hline $1,00 \times 10^{-4}$ & $2,8 \times 10^{-5}$ \\
$1,00 \times 10^{-3}$ & $8,9 \times 10^{-6}$ \\
$1,00 \times 10^{-2}$ & $2,8 \times 10^{-6}$ \\
$1,00 \times 10^{-1}$ & $8,8 \times 10^{-7}$ \\
$1,00 \times 10^{0}$ & $2,8 \times 10^{-7}$ \\
\hline
\end{tabular}

A adição de um eletrólito suporte em concentrações elevadas pode levar à adoção de modelos mais simplificados para descrever a interface. Por exemplo, o modelo de Helmholtz, que consiste em descrever a interface como um capacitor de placas paralelas, é utilizado na ausência de adsorção específica. Em outras palavras, admite-se apenas a camada compacta. Esta aproximação é feita, na dedução da equação de Butler-Volmer, descrevendo a dependência entre a densidade de corrente e o potencial, quando a reação de transferên- 
cia de carga é a etapa limitante na velocidade do processo de eletrodo. Um caso particular, para elevados sobrepotenciais, seria a equação de Tafel ${ }^{4,5}$.

\section{Manter a viscosidade da solução constante}

A viscosidade de soluções aquosas depende significativamente da concentração da solução. A presença de ES torna possível manter a viscosidade da solução constante durante grande parte dos processos eletroquímicos sob condição estacionária, mesmo na vizinhança do eletrodo, se mantida constante a temperatura, desde que as espécies eletroativas, reagentes e produtos, sejam solúveis. Entenda-se por condição estacionária, a condição obtida quando se utiliza convecção forçada, como por exemplo, empregando-se eletrodos rotatórios convencionais. Um exemplo de exceção, seria a oxidação de um metal produzindo espécies solúveis a elevadas densidades de corrente anódica, mesmo sob condição estacionária: a viscosidade da solução pode mudar, na região da camada de difusão, se a concentração da espécie, em virtude da sua alta solubilidade, for comparável ou maior que a concentração do eletrólito suporte.

\section{Manter o coeficiente de difusão da espécie eletroativa constante}

Admitindo o modelo de Stokes-Einstein ${ }^{5}$ o coeficiente de difusão (ou difusivididade) de uma espécie $i$ é dado pela Equação 9

$D_{i}=\frac{R T}{6 r \eta N}$

onde $r_{i}$ é o raio da molécula ou íon, admitindo a forma esférica; $\eta$, a viscosidade dinâmica da solução e $N$, a constante de Avogadro.

É fácil entender que a presença do eletrólito suporte, tornando $\eta$ constante, torna $D_{i}$ constante em processos isotérmicos.

\section{Manter constante o número médio de ligantes}

Muitas vezes há necessidade de se empregar um eletrólito cujo ânion é complexante para o íon metálico eletroativo. Nesse caso, a adição de um eletrólito suporte com ânion comum é importante para tornar constante o número médio de ligantes, mesmo na vizinhança do eletrodo. Um exemplo seria o estudo eletroquímico do par redox $\mathrm{Cu} / \mathrm{Cu}(\mathrm{I})$ em meio de cloreto. $\mathrm{O}$ emprego do cloreto de sódio como eletrólito suporte torna constante a composição da solução, à medida que $[\mathrm{Cu}(\mathrm{I})]<<\left[\mathrm{Cl}^{-}\right]$.

A manutenção do número médio de ligantes constante contribui, também, para a constância no valor de $D_{i}$.

\section{Aumento da condutividade da solução}

Muito embora a condutividade da solução possa ser elevada pelo aumento da concentração de qualquer eletrólito, eletroativo ou não eletroativo, no caso de se empregar um ES, sem dúvida esta também é uma de suas funções.

O ES será responsável por manter constante e alta a condutividade da solução, uma vez que a espécie eletroativa apresenta valor de $t_{i}$ muito baixo. A condutividade elevada tornará o termo RI pouco significativo. RI, denominado de queda ôhmica, é a mudança de potencial devida ao produto de corrente pela resistência da solução contida entre a superfície do eletrodo de trabalho e a do eletrodo de referência (ER). Em geral utiliza-se capilar de Luggin - Habber, para minimizar a queda ôhmica. A queda ôhmica, na presença de ES pode ser desprezível em um intervalo de várias ordens de grandeza de corrente, desde que as dimensões do eletrodo e da célula sejam adequadamente escolhidas.

\section{Efeito na cinética dos processos de eletrodo}

A literatura mostra, em grande número de trabalhos ${ }^{24-32}$, o efeito do eletrólito suporte nos valores dos parâmetros cinéticos de processos de eletrodo e nas características de depósitos metálicos. Este efeito tanto pode ser causado pela complexação dos íons metálicos por ligantes do eletrólito suporte, pela adsorção de ânions do eletrólito suporte ou, ainda, pela participação do eletrólito suporte no transporte de massa, reduzindo o transporte por migração do íon eletroativo. Por exemplo, a eletrodeposição de níquel é grandemente influenciada pela natureza do eletrólito suporte: a presença de íons cloreto, por formar complexos com íons $\mathrm{Ni}^{2+}$, torna os depósitos quebradiços, enquanto banhos contendo sulfato apresentam depósitos mais aderentes ${ }^{32}$. É bem conhecida a influência de eletrólito contendo cloreto, nos processos de corrosão. A ação do cloreto dá-se de várias formas: formação de complexos com íons metálicos ${ }^{21}$, adsorção na superfície do eletrodo ${ }^{33}$, hidrólise ácida dos cloretos dos metais de transição, com redução do pH na interface ${ }^{22}$. Em processos de adsorção, se o ânion do eletrólito suporte for complexante ao ponto de estabilizar o íon do estado de oxidação mais baixo, o produto de corrosão metálica pode ser diferente. No caso do cobre, por exemplo, a corrosão deste metal em meio não complexante, como sulfato, produz o íon $\mathrm{Cu}$ (II). Em meio de cloreto, por exemplo, como eletrólito suporte, este ânion não só afeta a cinética do processo como pode mudar o produto da oxidação, estabilizando o íon intermediário, o $\mathrm{Cu}(\mathrm{I})^{9,24,25}$. O efeito do eletrólito suporte sobre a reação $\mathrm{H}^{+} / \mathrm{H}_{2}$ vem sendo estudado desde a primeira metade do século XX, conforme descrito nos trabalhos de Frumkin et $a l .^{26}$. Neste caso, o principal efeito dá-se na modificação dos parâmetros cinéticos, em particular na densidade de corrente de troca e no coeficiente de Tafel, em virtude de modificações resultantes da adsorção dos íons presentes na estrutura da dupla camada elétrica.

Kruijt et al. ${ }^{27}$ mostraram o efeito do eletrólito suporte na velocidade de crescimento de depósitos de mercúrio sobre platina. Os autores observaram que a presença de $\mathrm{KNO}_{3} 1 \mathrm{~mol} \mathrm{~L}^{-1}$, em soluções contendo $0,05 \mathrm{~mol} \mathrm{~L}^{-1} \mathrm{Hg}_{2}\left(\mathrm{NO}_{3}\right)_{2}$ e $0,0001 \mathrm{~mol} \mathrm{~L}^{-1}$ de $\mathrm{HNO}_{3}$ modifica a cinética de nucleação do depósito, à medida que modifica os processos de transporte de massa e a queda ôhmica. Neste mesmo trabalho, os autores fizeram um tratamento teórico sobre a distribuição da velocidade de nucleação na vizinhança de crescimento de um "cluster".

Lacour et al..$^{28}$ estudaram o efeito da força iônica do eletrólito suporte sobre a cinética de adsorção da albumina, sobre eletrodo rotativo de carbono vítreo. Medidas de capacitância da dupla camada em função do tempo mostraram o efeito da força iônica sobre as constantes de tempo de adsorção. Tais efeitos foram atribuídos a três fatores: mudança da carga elétrica da interface, mudança da carga elétrica da proteína e mudança na espessura da dupla camada difusa.

Os estudos do efeito do ES sobre reações de eletrodo continuam merecendo destaque na literatura, como provam os trabalhos de Da Silva et al. ${ }^{29}$ sobre as reações de produção de oxigênio e de ozônio, e os de Huafang et al..$^{30}$ envolvendo o ferroceno e polieletrólitos. $\mathrm{O}$ efeito do eletrólito suporte sobre a atividade catalítica de eletrodos metal-óxido vem sendo objeto de estudos. De acordo com Zanta et $a l .^{31}$, a área eletroquímica ativa do eletrodo decresce com o aumento do tamanho do cátion do eletrólito suporte, tendo como exemplo os cátions presentes em $\mathrm{HClO}_{4}, \mathrm{LiClO}_{4}$ e $\mathrm{NaClO}_{4}$.

\section{Efeito no número de transporte na interfase polímero condutor - solução}

Conforme Maia et al. ${ }^{34}$, desde o início da década de 70 foi de- 
monstrada a possibilidade de preparar polímeros com propriedades semicondutoras. Após a metade desta década, descobriu-se que a condutividade poderia ser bastante incrementada pelo processo de "dopagem". Os polímeros, com modificações químicas apropriadas, podem mostrar condutividades desde semicondutores até condutividades da ordem da do cobre. Das famílias poliméricas com propriedades condutoras, as mais estudadas são as do poliacetileno, polianilina, polipirrol e politiofeno. Tais polímeros apresentam ampla variedade de aplicações tais como baterias plásticas, superfícies condutoras, compósitos condutores, diodos emissores de luz (LED) etc.

Trabalhos mais recentes têm mostrado a importância do ES nas interfases eletrodo - solução, quando na presença de polímeros condutores. Borole et al. ${ }^{35}$, por exemplo, estudaram o efeito de diferentes eletrólitos inorgânicos ácidos (ácidos sulfúrico, clorídrico, nítrico, fosfórico e perclórico) e orgânicos ácidos (ácidos benzóico, oxálico, malônico, succínico e adípico) nas propriedades eletroquímicas de eletrodos modificados por diferentes polímeros condutores, como a polianilina (PA), a poli-ortotoluidina (POT) e o co-polímero PA-POT. Empregando voltametria cíclica estes autores observaram que as densidades de corrente são fortemente influenciadas pela natureza do ânion, bem como a condutividade na fase polimérica.

\section{CONCLUSÕES}

O eletrólito suporte tem ampla aplicação em processos de eletrodo e, a princípio, deve apresentar como propriedades, alta solubilidade, alto grau de ionização, estabilidade química e eletroquímica. Com relação às suas aplicações, verifica-se que são bastante amplas: mantém os coeficientes de atividade praticamente constantes, o número de transporte da espécie eletroativa praticamente igual a zero, diminui a espessura da dupla camada elétrica, mantém a viscosidade, o coeficiente de difusão e o número médio de ligantes ( no caso de ser o ânion um complexante) constantes, além de incrementar a condutividade em meios de solventes polares, tanto orgânicos quanto inorgânicos.

Por outro lado, cuidados devem ser tomados e critérios devem ser adotados na escolha de um eletrólito suporte. A sua presença, por exemplo, modifica as propriedades das soluções e das interfaces eletródicas, causando efeitos na termodinâmica e na cinética tanto de processos de transferência de carga quanto de adsorção na superfície do eletrodo.

Os critérios para emprego do eletrólito suporte são diferentes quando se empregam micro e ultramicroeletrodos, no que se refere à correção da queda ôhmica.

A escolha do eletrólito suporte deve levar em conta a natureza do substrato empregado como eletrodo, bem como a natureza da investigação a ser realizada. Cabe ao pesquisador nortear a escolha em função das particularidades do sistema em consideração e dos objetivos do seu trabalho.

\section{REFERÊNCIAS}

1. Delahay, P.; Double Layer and Electrode Kinetics, Wiley: New York, 1954.

2. Brett, A. M. O.; Brett, C. M. A.; Electroquímica. Princípios, Métodos e Aplicações, Oxford University Press, 1993.

3. Ticianelli, E. A.; Gonzalez, E. R.; Eletroquímica, Edusp, 1998.

4. Koryta, J.; Dvorák, J.; Kavan, L.; Principles of Electrochemistry, Wiley: England, 1993

5. Bockris, J. O'M.; Reddy, A. K. N.; Modern Electrochemistry, Plenum Press: New York, 1977.

6. Oldham, H. B.; Myland, J. C.; Fundamentals of Electrochemical Science, Academic Press, Inc, 1994.

7. Plambeck, J. A.; Electroanalytical Chemistry, Wiley, 1982.

8. Hamann, C. H.; Hamnet, A.; Vielstich, W.; Electrochemistry, Wiley, VCH: Weinheim, 1998.

9. Agostinho, S. M. L.; Dissertação de Mestrado, Instituto Tecnológico de Aeronáutica, Brasil, 1971.

10. Sawyer, D.T.; Sobkowjak, A.; Roberts, J. L.; Electrochemistry for Chemists, $2^{\text {nd }}$ ed., Wiley: New York, 1995.

11. Wang, J.; Analytical Electrochemistry, $2^{\text {nd }}$ ed., Wiley: New York, 2000.

12. Rieger, P. H.; Electrochemistry, $2^{\text {nd }}$ ed., Chapman \& Hall, 1993.

13. Gileadi, E.; Electrode Kinetics, VCH Publishers, Inc.,1993.

14. Lingane, J. J.; Electroanalytical Chemistry, Interscience Publishers, Inc., 1958.

15. Atkins, P. W.; De Paula, J.; Physical Chemistry, $7^{\text {th }}$ ed., Oxford University Press, 2002.

16. Castellan, G.; Fundamentos de Físico-Química, LTC-Livros Técnicos e Científicos Ed. S.A., 1996.

17. Oldham, K. B.: J. Electronal. Chem. 1988, 250, 1.

18. Hyk, W.; Palys, M.; Stojek, Z.; J. Electroanal. Chem. 1996, 415, 13.

19. Lobo, V. M. M.; Portugaliae Electrochimica Acta 1996,14, 27.

20. Bjerrum, J.; Schwarzenbach G.; Sillen, C. G.; Stability constants, Part II: Inorganic Ligand, Chemical Society, Burlington, House: London, 1958.

21. GEEQuim-Grupo de Educação e Ensino de Química; Experiências sobre Equilíbrio Químico, Instituto de Química, USP, Brasil,1985.

22. Bard, A. J.; Faulkner L, R.; Electrochemical Methods. Fundamentals and Applications, $2^{\text {nd }}$ ed., Wiley: New York, 2001.

23. Mohilner, D. M. Em Electroanalytical Chemistry; Marcel Dekker Inc: New York, 1966.

24. Costa, S. L. F. A. da; Agostinho, S. M. L.; Chagas, H. C.; Rubim, J. C.; Corr. 1987, 43, 149.

25. Costa, S. L. F. A. da; Rubim, J. C.; Agostinho, S. M. L.; J. Electroanal. Chem. 1987, 220, 259.

26. Frumkin, A. N.; Bagotsky, V. S.; Iofa, Z. A.; Kabanov, B. N.; Kinetics of Electrode. Processes, Izd. MGU, 1952, apud Antropov, L.; Theoretical Electrochemistry, trad. Artavaz Beknazarov, Mir Publishers: Moscou, 1977.

27. Kruijt, W. S.; Sluyters-Rehbach, M.; Sluyters, J. H.; Milchev, A.; J. Electroanal. Chem. 1994, 371, 13.

28. Lacour, F.; Ficquelmont-Loizis, M. de; Caprani, A.; Electrochim. Acta 1991, $36,1811$.

29. Da Silva, L. M.; De Faria, L. A.; Boodts, J. F. C.; Electrochim. Acta 2003, 48,699 .

30. Huafang, Z.; Shaojun, D.; Electrochim. Acta 1997, 42, 1801.

31. Zanta, C. L. P. S.; de Andrade, A. R. A.; Boodts, J. F. C.; Electrochim. Acta 1999, 44, 333.

32. Panossian, Z.; Superfície, ABTS, 1997, 20.

33. Agostinho, S. M. L; Tese Doutorado, Instituto de Química, Universidade de São Paulo, Brasil,1975.

34. Maia, D. J.; De Paoli, M. A.; Alves, O. L.; Zarbin, A. J. G.; das Neves, S.; Ouim. Nova 2000, 23, 204

35. Borole, D. D.; Kapadi, U. R.; Kumbhar, P. P.; Hundiwale, D. G.; Mater. Lett. 2002, 56, 685 . 